\title{
Comentario sobre 154 casos de prolapso genital
}

Doctor ARTURO APARICIO IARAMMLO

Profesor Titular Clínica Ginecológica - Bogotá.

Trâbajo presentado al il Congreso Nacional de Cirugía. - Medellín.

En el año 1942 presentamos a la consideractón de la Socledad de Cl. rugia de Bogotá, un trabajo titulado -A Prolapeo Gentiols. Consideracio nes Anatómicas y Fisio-pátológicas del Prolapso y su tratamiento por la operación de Halban publicado en enero de 1942 en el eRepertorio de Medicina y Cirugía de Bogotá. En ese entonces, tratamos de agotar el tema on materia ton importante como la anatomía del piso pélvico: revaluamos los conceptas en relación con los elementos que sostienen y suetenton la matriz; estudiamos su estática y de jamos aclarados los problemas relacionados con su etio-patogenia, con el exclusivo objeto de sentar las bases de un tratamiento quirúrgico, que, como la operación de Halban cumpliera con los preceptos anatómicos, flisiolé. qloos y tunetonales de la modema Git necoloric.

Como basta la lecha no se han mo. diflacodo ni desvirtuado tales concep. tos, por lo demás, hoy de todos los enpecialistas conocldos, nos hemos 11 . mitado en el presente trabajo, $\alpha$ botudiar culdadosamente 154 abservacto. nes tóthades de nuestro archivo per. sonal y comprendidas entre 100 ahos de 1943 y 1951, thaluativo, delando de lado toda consideración documental ya sea bibliogréfiloa o académica. Vo luntariamente tambtén, hemos estudiado ton sólo esas historias independientemente del trabajo de Hospital, para no dar a este Congreso la tan conocida disculpa en nuestro medio. de falta de control. En la clientela pri vada, por múltuples razones de orden social y oconómico, el control es más covero, más alustedo a la realidad Y - ojercs indiscuttblomente tanto do parte del cirujano como de parte del paciente, quien no vacilará ante un fracaso, de presentar un justo recla. mo.

Con estas salvedades, presento pues, a la consideración de esle Congreso, el resultado de mis obeervactones en el curio de estoe uiltmoe 9 añne on forme de cuevitros est

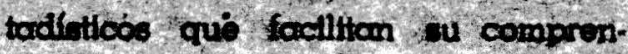
dín, arregando alounos comentarios resultantes do su cetudio y de nuevtra experiencia perconal, que didá pu. diercon eer útiles en al descrrollo del tan discutido problema del . Tratomiento del Prolopeo Gentials. 


\section{CONSIDERACIONES GENERALES}

Seqún nuestro criterio, que no se ha modificado desde nuestra primera publicación a que hicimos referencia, el eProlapso Genital debe ser considerado como una hernia visceral a través de un punto débil, en cuyo caso el orificio herniano estaría constituido por el hiatus uro-genital, ensanchado por la relajación o desgarradura de los manejos puborectales del elevador. No obstante esta perentoria afirmación, es necesario tener en cuenta que la patogenia del prolapso no es tan simplista como a prime. ra vista parece y que los órganos pueden prolapsarse individual 0 conjuntamente de acuerdo con el trauma - grado de agresión recibido. Más explícitamente: un desgarro del perineo, desgarro de tercer grado, por ejemplo, no produce, como consecuencia obligada, un descenso de la pared anatómica de la fascia vésico-vagi. na]. La inversa, es igualmente evidente y de esto podemos deducir lo pro teiforme de sus variedades y por consiguiente la variedad de las técnicas que debemos seguir para tratar cada caso en particular.

No obstante estas ideas, conceptuamos en tesis general, que el prolapso genital debe ser tratado continuando los principios generales seguldos en cirugía para el traiamiento de las hernias y que los tiempos operatorios de la cura radical y completa de la Hernia Genital deben llenar al menos el mínimum de requisitos exigidos en tales casos, vale decir, estrechando el Hiatus o Hendidura genital; rehacer cuidadosamente el aparato de sostén (periné y fascias) y por último dos- truír hasta donde sea posible, la co rrespondencia de los ejes uterino y vaginal. Por esta razón, técnicas que no cumplan a cabalidad con estos re quisitos, serán siempre defectuosas; La Histeropexia, por ejemplo, mejora el descenso uterino pero no corrige el prolapso vaginal, ni rehace el suelo perineal. La histerectomía vaginal. tan aconsejada por algunas escuelas americanas, no es de nuestro personal agrado, entre otras razones, porque al destruír el órgano, destruye una función en contra de todos los principios fisiológicos anotados ante riormente y de otra parte, porque la experlencia nos ha enseñado que el mayor número de casos encontrados en nuestras dos estadísticas, fluctúan entre los 31 y los 40 años, época de la mayor actividad sexual de la mujer. No quiere esto decir, que la Histerectomía vaginal, como medio de excepción no tenga para nosotros sus indicaciones $y$ asi lo hemos practica. do en algunos casos, en donde lesio. nes sobre agregadas que puedan pre. sentarse concomitantemiente con ef prolapso, aconsejen ese tipo de intervención.

Con las técnicas quirúrgicas, que bien podríamos llamar modemas, de rivadas todas ellas de la Clásica Opo ración de Halban, así se llamen: Manchester, Donald o Fothergill, etc. se cumple a cabalidad, segrín nuestro criterio, con el objetivo primordiat del tratamiento racional de la Hernia Geni. tal y a ellas hemos seguido adictos en el curso de estos últimos 10 años. Hemos dejado de lado un tanto la inteivención de Halban, por ser de eje cución más engorrosa y sólo la practicamos a título académico, euando 
deseamas demostrar ante los alumnos lic existencia de las tascias. Es más rápido, quizás más elegante.. el practicar el repliegue vesical y dar sustentación a la vejiga por medio de los ligamentos pubo-vesicales. En este tiempo, casi siempre lo comple mentamos aplicando unos puntos de reluerzo a da manera de Kennedy so bre el cuello vesical, con el objeto de corregir la incontinencia de estuerzo. complicación por demás harto trecuente en el descenso vesical. La carrec. ción de la retroflexión la practicamos cuando existe, siguiendo las dos variantes de la operactón de Halban. que merecen ser brevemente recordadas: la primera variante se relaciona con el tamaño total de la matriz, y así. cuando se encuentra un tamaño mayor al de $8 \mathrm{cms}$. lo que aquivale decir que el cuello está hipertrofiado. ejecutamos la amputación del cuello. calcuiundo que la matriz quede de un tamaño aproximado de $7 \mathrm{cms}$. La retro-desviación uterina, se corrige en parte en esta variante, medicante la neo-implantación de los ligamentos cardinales o transversos de Macken. rodt, por encima de la sección del corte cervical y de otra parte, aplicando hilos de sutura que pasion por el itsmo evitando los espacios muertos y dejando a esa altura un fuerte y denso tejido de cicatrizaclón. Las pocas veces que no hemos amputado el cuello, hemos dobtalo lomentarlo, por presentarse algunos años después, la recidiva del pro lapso corvical. (DOs casos dentro de nuestra casuística). Hecho importante que debemos recordar al pracHicar la amputación del cuello, es la. faclildad con la cual puede ocluirse parcialmente el ortucio cervical, pot una exagerada coaptación de los col. gajos vaginales. Para evitar dicha eventualidad, aconsejamos dejar sisto máticamente, como es nuestra costumbre, o bien un tallo rígido de plata, tipo Iribarne, o simplemente un caucho rígido que se retirará oportunamente a los 8 o 10 dias.

La segunda variante o modalidad de Halban, la practicamos cuando la matriz es de un támaño menor al de $8 \mathrm{cms}$. y necesitamos corregir la lle dón uterina Camo se recordand, esta variante consiste en corregir la retro verstón por medio de la véstcafliar clón alta, obliterando el espacio vé sico-uterino, lo que hace imposible la interposición del intestino entre la vejiga y el útero. Para practicar esta maniobra, es necesario abrit ppeviamente el fondo de saco vésico-utertno, luxar el fondo del útero y suturar la porción ftia del peritonio vesical a la cara posterior del fondo del útero. Esta variante al elecutarse con alouna prócilca, relativamente sench lla, absolutamente inocua y do cicuer do con nuestra estadistica da un margen de seguridad del $100 \%$, en relación con la cura de la retroflexión. No podemos decir lo mismo acerca del resultado obtenido en la cura de la retroflexión por medio de la sim. ple amputactón del cuello, pues en nuestros controles bemos encontrado un aprectabio númoto de corbs in los que, pese a la integricad de la escatriz operatoria, homos hallado of cuerpo on flextones vartables do 0 gundo y tercor grados.

En muchos casos de prolapso, más de lo que puede creerse generalmen. to. exdeto una liernia del fondo do 
saco recto-uterino, observándose en alqunas ocasiones verdaderos enteroco les, lo que obliga a escindir el perito neo posterior con objeto de cerrar el fondo de saco de Douglas, mediante sutura con seda, de los ligamentos útero-sacros, maniobra que por parte ayuda a corregir la flexión. Para terminar la intervención $y$ cualquiera que sea la variante que hayamos empleado, practicamos invariablemente una perineoplastia con miorratia de los elevadores.

Como se podrá observar por la téc nica quirúrgica que a grandes rasgos acabamos de anotar, usamos en nuestros casos una mezcla de las operaciones de Halban, de Fothergill $y$ de Manchester, resultante de nuestra experiencia y con miras exclusivas, de conservar íntegramente las funciones fisiológicas de los órganos comprometidos, no sólo en lo que a la menstruación se refiere, sino también al embarazo, parto y puerperio. como lo demuestron algunas de nuestras observaciones. Para la comodidad de nuestra estadística la llamaremos Fothergill Manchester, haciendo hincaplé una vez más, que en materia de selección de técnicas para la cura del prolapso, ninguna de ellas es perfecta y que en muchas de nuestras observaciones hemos tenido que recurrir a procedimientos combinados (via abdomino-vaginal) para poder resolver de una manera satisfactoria, determinados problemas del prolapso. De todas maneras, y cualquilera que sea la técnica escogida il queremos obtener buenos resultados, debemos cimentarla en un conocimiento profundo de la cmatomía de la región; en la eecurided $y$ maestría con que aprendamos a manejar y a disecar las fascias y ligamentos importantes y en el conocimiento perfecto de los principios que rigen la etiopatogenia del prolapso.

\section{RESULTADOS $Y$ COMENTARIOS}

\section{Cuidados pre y post-operatorion.} Con el advenimiento de la suliamidoterapia y de los antibióticos, hemos modificado substancialmente nuestra conducta en relación con el Pre y el Post-operatorio. Mientras que antaño teníamos especial cuidado en prepa. rar la mucosa vaginal para la inter. vención por medio de duchas vaginales salinas o ácidas, y preparábamos la enferma desde dos días antes de la intervención mediante la administración de laxante aceitoso y constipación subsiguiente por medio de opićceos, hoy nos limitamos exclusivamente a curar los cuellos ulcerados, mediante reposo y aplicación de mechas embo bidas en una mezcla de aceite de Hígado de Bacalao y sulfas o mechas a la glicerina ictiolada. En los demás casos no hacemos tratamiento alguno especial, excepto que aconsejamos en veces baños de asiento emolientes con manzanilla o administración de estrógenos ya sea en aplicación local (vaginal) o simplemente por vía enieral. Los cuidados que cas no he mos variado, son aquellos referentes al post-operatorio, en los que a más de administrar antibib́ticos cada 12 horas durante 304 días, practicamos duchas vaginales con solución salina a partir de las 24 horas sigulentes a la operación, momento en el cual re thromos la mecha aceltosa y empa. 
pada en sulfa que colocamos siste máticamente al finalizar toda intervención varginal. Dejamos siempre sonda de Folly a permanencia por 3 a 5 días que retiramos una ver que el paciente haya evacuado artificial o espontánearnente su intestino. Levanta. mos la paciente invariablemente entre el cuarto $y$ el quinto día, do la intervención.

Como material de sutura empleamos sistemáticamente Catgut delgado números 0 o l y cromado delgado pára las suturas de las fascias y de los-elevadores. Casi nunca usamos material no absorbible.

Inestesia. Usamos en nuestra clientela privada casi exclusivamente pentotal cicloéter, excepto en las enfermas de edad avanzada en cuyo caso empleamos siempre anestesia local, con magníficos resultados. La raquianestesia sólo la usamos como método de excepción.

Inditis do cusadros sitadieticon Ro visando nuestra estadistica cotud, que para comodidad de su estudio hemos dividido en cuadros estodísticos, observamos que sus resultados son muy si. milares a los presentados en nuestro primer trabajo. Analizando estos cuadros obteniemos el siguiente resultado: 1. Martalidad. Sobre 154 casos ope rados en la última década, tuvimo dos muertes que lamentar, lo que da en nuestra eatadistica un porcentaje de $1.29 \%$ de mortalidad. La primera de las muertes se debló a shock anes. tésico, por raquicanestesta a la media hora de la intervención y el segundo - deceso se produjo a los 15 días do la intervención, súbliamente, posiblemen. to por iniarto candíaco.
CUADRO I

Edod do las conruden

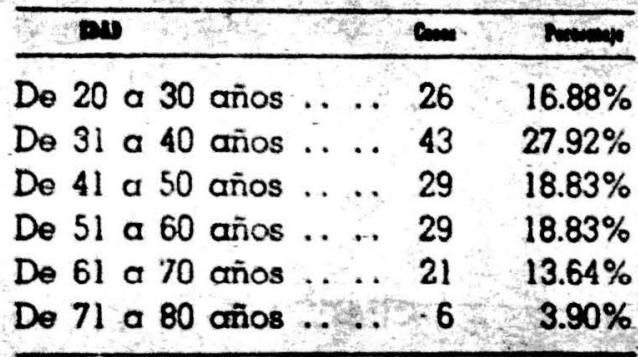

Comentada La edad más trecuente ha sido la comprendida entre las 31 a los 40 años (27.92\%); hecho tebre of cual llamamos nuevamento la atención, pues demuestra claramente, que la mayor cifra o porcentaje de prolapsos, corresponde a la época de completa madurez sexual en ia mujir. De donde podemos deciucir yue la terapéuttca quirúrgica, debe ser errinontemente conservadora $y$ le cecuerdo con los principlos anotomo-fisilógicos 80 bre los cuales no nos canscremos de instats anto nueatros dincipulos Co mo dato complementarto merres ano tarse el hecho de que las pacientes operadas entre los 20 y los 50 años (98 casos), presentaron embarazos ulteriores con parto a término, 8 pacientes, lo que da un porcentale no des. preciable del $8.36 \%$. De 2 ctos casos, sólo uno presentó recidiva, curactert zada por descenso a hipertrolia corvh cal con rolajactón del pito pertnect. lo que obligó a practicar camputación del cuollo y nueva plantia perined. Los demćs casos, no presenteron to. cidiva alguna, y lodos ellos de celer. do con el informe de los tocólogos aue los atendieron, no presentaron $0 \mathrm{~m}$ plicactón y la dilatactón cervical so 
efectuó normal y espontóneamente. aún en aquellos casos en que se había practicado-la amputación cervical. Recalcamos una vez más, en la necesidad de practicar cuidadosamente esa amputación, ojalá sobre tallo rígido como la ejecutamos, con el objeto de modelar convenientemente el cuello y el orificio cervical.

\section{CURDRO II}

\section{Partos}

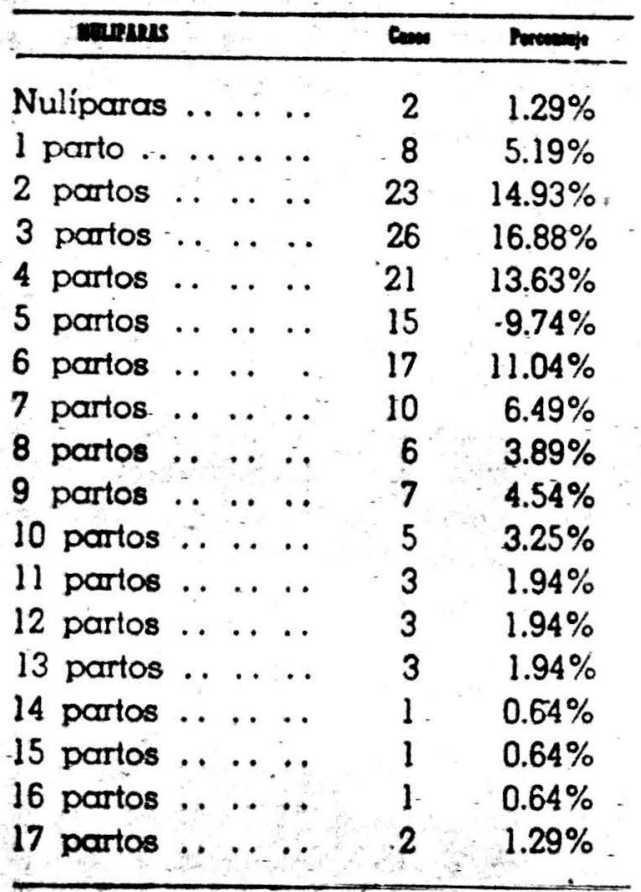

Cononicerio. Como podemos observar del estudio atento de este cuadro. el mayor porcentaje se encuientra en mujeres que han tenido de 2 a 6 partos $(64.22 \%)$, siendo menor el porcentaje de las grandes multíparas. Entre el oran total de 154 entermas, única. mente pudimos encontrar que sólo a 12 de ellas, se les había aplicado fórceps, casi siempre en el primer parto (7.07\%). Los demás casos anotan lás pacientes partos más o menos laboriosos y levantamientos precoces. Sin darle a este último hecho una importancia definitiva en la patogenia del prolapso, queremos hacer resaltar su evidencia y llamar la atención de los médicos que lo aconsejan de una manera Indiscriminada. El hecho que el mayor número de Prolapsos se encuentra en pacientes, que han tenido de 1 a 4 hijos y que carsi siempre es consecutivo al trauma del primer parto, significa en nuestro modo de pensar, que no es la gran multiparidad la causa del descenso, sino simplemente la agresividad con la cual se efectúa el parto, sin olvidar la constitución o debilidad de los tejidos variables en cada caso en particular. De acuerdo con estos factores, el médico tratomie podrá o no, ordenar el levantamiento precor de su paciente. Nunca sistematizarlo, como es la tendencia actual de muchas escuelas.

De otra parte, es muy frecuente en nuestro medio, en no dar mayor importancia a la correcta reparación de] periné, limitándose en muchas ocasiones a la-simple sutura de la mucosa desgarada. Creemos firmemente que si esos perinés, se trataran más con criterlo quirúrgico que obstétrico, vale decir, reparando convententemente 108 elevadores que por tuerza del parto se encuentran dilascerados o simplo mente separados, veriamos disminuír de una manera notoria, la incidencla del prolapso. 


\section{CUADRO III}

\section{Sindomatoloria}

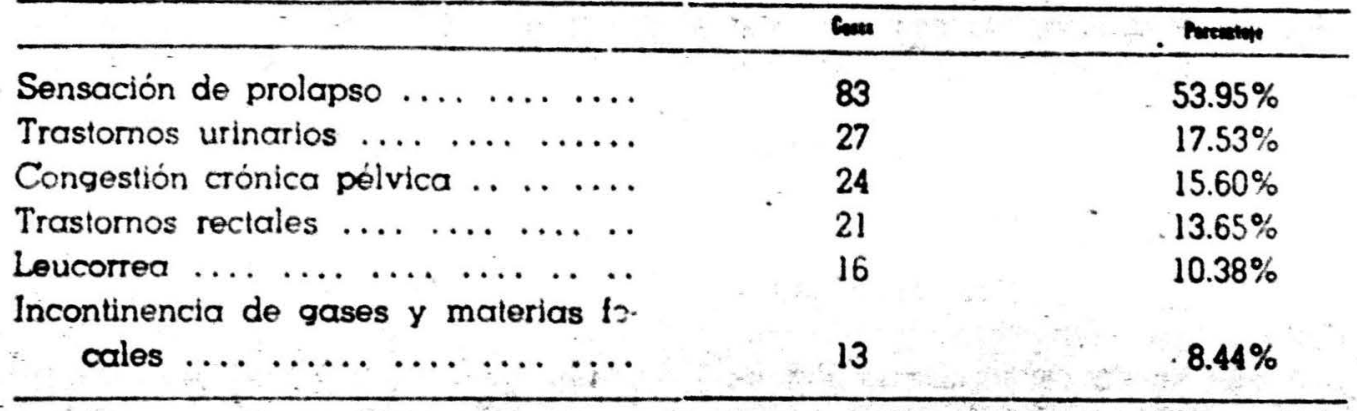

Comentario. En este cuadro hemos tomado los sintomas dominantes, pero existen algunos casos en los que lógicamente, la paciente se aqueja de uno o dos síntomas, por ejemplo, sensación de prolapso y trastornos urinarios; congestión crónica y leucorrea, etc. Por esa razón, la suma de los casos y la de los porcentajes, aparece mayor a la suma total de tós casos presenta. dos. Hecha esta salvedad, podemos observar en tesis general, que los sín. tomas clínicos observndos por las paclentes, están en relación directa con la modalidad o variedad del prolapso $y$ de acuerdo con el brgano predomlnantemente prolapsado. No es lo mismo la sintomatología presentada por una enferina que siente permanente mente entre sus genitales externos un cuello uterino prolapsado, a aquélla, que sólo presenta un descenso vesical - rectal. Para la primera, la molestla dominante sert la senscrión de peso: para la otra, la molestia será de tipo vesical o rectal. En nuestra casuistica, hemos agrupado un número no dos preciable de casos (24), cuyo sintoma dominante ha sido el cuadro de Congestión Crónicas, imputable en un principio a la marcada retroflexión, poro quie a la exploración operatoria dis minuye en importancia ante las lesio nes concomitantes encontradas: salpin. gitis crónicas, varicocele pélvico, endometriosis, etc.

De esta variedad de sintomas, podemos deducir la importancia que para el especialista tiene el estudio mlnucioso de cadr cosso en particular. para poder orientar con un criterio de sana lóvica el tratamiento. No es posible, eovín nuestra manera de pen: sar, siatematizar una técrica on materia de tratamiento tel prolapso, nl mucho menos seguir una línea de con. ducta rigida que nos permita seguir estrictamente determinada técnica o escuela. El especialista debe tener on sus manos, un acervo de conocimientos y de técnicas por aí dectrlo, que le permita un cada cono sortear con facilidad ol problema y benoliciar ai pactente. Por esa rarón, cuando se me pregunta cuál es la tbenica de mi pre. dilección para la cura del prolapso. invariablemente contesto: Técnica e8pecial, no tengo: aplico en cada caso. la técnica o técnicas que sean necesarias para soluejonar el problema. 
En nuestra estadística hemos agrupado los casos en prolapsos de prime ro, segundo y tercer grados, de acuerdo con el descenso uterino, acompanados casi siempre de cisto-rectocele y desgarro perineal variable. Presentamos 3 casos de prolapso uterino exclusivo en vírgenes, con marcado alargcmiento hipertrófico del cuello y relajacián del piso perineal, pero sin cisto o rectocele. Vienen luego las va. riedades de colpo-cistocele o colpo. rectocele, sin descenso uterino alguno; el cisto-rectocele y el desgarro de tercer grados que los hemos clasificado como prolapsos parciales y finalmente hemos hecho una última agrupación, constituída por aquéllos casos en que el hallazgo dominante es una marcada retroflexión y el síntoma do minante un cuadro de congestión pélvica, lo que nos ha obligado a seguir una línea de conducta mixta: vaginal, para tratar el cisto-rectocele y abdo minal, para tratar la retroflexión y a algunas lesiones concomitantes.
Comentario. Analizando este cuadro, podemos observar que la mayor frecuencia corresponde a las variedades clasificadas como de segundo y tercer grados (80 casos), pero nos ha sorprendido el hecho de encontrar un número no despreciable de casos (2I). en que el síntoma dominamte ha sido la congestión pélvica, imputable al grado de retroflexión. lo que nos ha obligado a variar nuestra línea de conducta quitrúrgica. No quiere esto decir, que en los demás casos no hayamos encontrado más o menos evidencia de retroflexión $y$ que al intervenír no hubléramos tratado de carre girla. Pero, el hecho de haber encontrado en nuestras pacientes, lesiones concomitantes que nos explicaran el cuadro de congestión, tales como: varicocele, salpingitis quisticas, ovaritis. endometriosis, etc., nos sugiere algunas reflexiones en relación con la importancia de las retrodesviaciones en la etio-patogenia y la sintomatología del prolapso. En efécto: st las retro-

\section{CUADRO IV}

\section{Modalidad dal prolapeo}

Prolapso genital de $1^{\circ}$ grado .......

Prolapso genital de 29 grado ....... Prolapso genttal de 39 grado ....... Prolapso uterino exciusivo (vírgenesi) .

Prolapso percial:

a) Colpo-dstocele simple ......

b) Colporectocele imple ......

c) Cisto-rectocele simple ... ....

Desgarro perineal de $\$$ grado .. .. Retrotexión de 39 grado con cisto-

\section{Cata}

\section{7}

38

42

3

9

8

13

13

$5.08 \%$

$5.01 \%$

$8.44 \%$

$8.44 \%$ 
desviaciones constituyeran una cousa preponderante en la etio-patogenia del prolapso, como hasta la fecha to ho mos aceptado, mayor debería ser al número de prolapsos, ya que es inusitada la cantidad de retrodesviaciones asintomáticas encontradas en la diaria consulta y $\sin$ evidencia alguna de descenso. De otra parte, las técnicas quirúrgicas, tipo Manchester. debieran ser revisadas en relación con la corrección de la retroversión, ya que en los controles cuidadosos $8 \theta$ -puede observar, la frecuencia de la reincidencia de la retrodesviación.

En lo que atañe a nuestra experien. cia y hablando honradamente, podemos decir, como lo expresamos al inicior este trabajo, que para corregir la retrodesviación hemos empleado las dos modalidades de la clásica Operación de Halban, es decir, la fijación alta de los ligamentos transversos con aplicarión de puntos de refuerzo 80 bre el istmo y la vésica-fijación alta, cuyos resultados nos han parecido infinitamente más satidactorlos. Pues blen: en las pacientes tratadas por la primera modalidad o variante, hemos observado en un alto porcentaje, la reincidencia de la retroflexión, sin observar empero, compromiso alguno en cuanto a la técnica de la intervención se refiere, y sin experimentar la enferma molestia alouna, lo que nos obllga a calificarla como de resuliado complia mente satisfactorio. Qulece ento dectr. que no podemos caeptar a ojos entrados que con eato tpo do Henicá o con el simple Manchester, corrijamos la retroflexión. Y si la enterma, no expe rimenta molestia alguna y no 80 pro duce la reincidencta del prolapso gent. tal, a pesar de esa mala posiclón de la matriz, debemos concluír que la ro trodesviación en si, no es una causa tan importante en la etiopatogenia del prolapso, y que, mientras los elementos musculares y tejidos que contribuyen a formar la hendidura uragenital se conserven intactos 0 se hayan so parado convenientemente, no se pro ducirá el prolapso genital. De sobra queda demostrado, la imporiancla del pertné para que hagamos hincapié en su correcta reparación, cualquiera que sea la técnica empleada en la corrección del prolapso genital.

Volviendo a nuestro punto de partida, es decir, los 21 casos anotados anteriormente, concluímos que sólo on el $13.64 \%$ de nuestros casos, dimos importancia a la retroflexión dentro de la variedad de prolapso, como para obllormos a variar de criterio quiríralca. parcentade que 20 desvirtía anto ol hecho de haber encontrado cast stempre la causa que nos explicara el síntoma de congestión. Este hallazgo, nos ha sugerido las presentes reflexiones, que nos llevan a pensar, que on rea. lidad no debemos dar mayor tmpor. tancia a la retroflexión como causa directa en la producelón del prolapeo y que las cuadros do congestión p4t ver pueden ter impulaibies a íctiones asnocmitantes y no al stmple hecho ds la rotroliedín. 
Imamente posemos concluir que siendo tan variada la modalidad del prolapso, es igualmente proteiforme la técnica que se debe emplear, y que en ningún caso podemos sistematizar una técnica al hablar de la cura del pra lapso genital. Parodiando el viejo afo rismo. podemos decir, que no existen prolapsos, sino enfermas de prolapso. y que cada caso en particular, debe ser sometido a culdadoso estudio para determinar cuál ha de ser la in. tervención que en nuestro criterio produzca los mejores resultados.
Vol. 1, noviembre 1y44, Lstula que se produjo en el momento de pasar los hillos sabre los ligamentes puba-vesica. les, con el abjeto de tratar el cistocole. Después de aclarar el diagnóstico, nos vimos precisados a practicar una neo. implantación del uretere, afortunadamente con muy buenos resultados. Desde entonces, tenemos siempre en mente esta posible complicación y especial cuidado en el momento de ese acto operatorio. Las demás complicaclones se ham tratado de acuerdo con los preceptos dásicos, pero vaio la

\section{CUIDAO VI}

\section{Complicaciones operatorias I pot-operatorian.}

Ruptura vesical (sin consecuencias .. Ruptura rectal (sin consecuencias) ... Fistula uretera-vaginal (caso publicardo) Incontinencia de esfuerzo ... . . . . Cistitis .................... Retencib́n de orina ........... Hemorragia post-operatorla ... .....
1

1

1

9

13

8
$0.64 \%$

$0.64 \%$

$0.64 \%$

$5.08 \%$

$8.44 \%$

$5.19 \%$

$5.60 \%$
Comentario. La más dramática de nuestras complicaciones fue la producción de una fístula uretero-vaginal del lado izqulerdo, que apareció el 129 día de la intervención. Sobre este caso de real interés, hicimos una publica. clón en la Boviala Cíniea, número 3. pena conotar que en esta estadistica no hemos encontrado ningún carso de dohiscencia del periné por hematoma o supuración, imputables a la buena cocptactón de las suturas y al emples sistemático de los entibióticos. 